\title{
Angiogenesis and vasculogenic mimicry as therapeutic targets in ovarian cancer
}

\author{
Dansaem Lim ${ }^{1}$, Yeojin Do ${ }^{1}$, Byung Su Kwon ${ }^{3}$, Woochul Chang ${ }^{4}$, Myeong-Sok Lee ${ }^{1,2}$, Jongmin Kim ${ }^{1,2, *}$ E Jin Gu Cho ${ }^{1,2, *}$ \\ ${ }^{1}$ Division of Biological Sciences, Sookmyung Women's University, Seoul 04310, ${ }^{2}$ Research Institute for Women's Health, Sookmyung \\ Women's University, Seoul 04310, ${ }^{3}$ Department of Obstetrics and Gynecology, Pusan National University School of Medicine, \\ Biomedical Research Institute, Pusan National University Hospital, Busan 49241, ${ }^{4}$ Department of Biology Education, College of Education, \\ Pusan National University, Busan 46241, Korea
}

Tumor angiogenesis is an essential process for growth and metastasis of cancer cells as it supplies tumors with oxygen and nutrients. During tumor angiogenesis, many pro-angiogenic factors are secreted by tumor cells to induce their own vascularization via activation of pre-existing host endothelium. However, accumulating evidence suggests that vasculogenic mimicry (VM) is a key alternative mechanism for tumor vascularization when tumors are faced with insufficient supply of oxygen and nutrients. VM is a tumor vascularization mechanism in which tumors create a blood supply system, in contrast to tumor angiogenesis mechanisms that depend on pre-existing host endothelium. VM is closely associated with tumor progression and poor prognosis in many cancers. Therefore, inhibition of VM may be a promising therapeutic strategy and may overcome the limitations of anti-angiogenesis therapy for cancer patients. In this review, we provide an overview of the current anti-angiogenic therapies for ovarian cancer and the current state of knowledge regarding the links between microRNAs and the VM process, with a focus on the mechanism that regulates associated signaling pathways in ovarian cancer. Moreover, we discuss the potential for $\mathrm{VM}$ as a therapeutic strategy against ovarian cancer. [BMB Reports 2020; 53(6): 291-298]

\section{INTRODUCTION}

Gynecologic cancers that occur in female reproductive organs include ovarian cancer, uterine cancer, vaginal cancer, cervical cancer, and vulvar cancer. According to data released in 2019,

*Corresponding authors. Jin Gu Cho, Tel: +82-2-710-9553; Fax: +82-2-2077-7322; E-mail: jgcho84@gmail.com; Jongmin Kim, Tel: +82-2-710-9553; Fax: +82-2-2077-7322; E-mail: jkim@sookmyung. ac.kr

https://doi.org/10.5483/BMBRep.2020.53.6.060

Received 20 March 2020

Keywords: Angiogenesis, Ovarian cancer, Therapeutic target, Tumor vascularization, Vasculogenic mimicry ovarian cancer has the highest incidence and mortality rate among gynecologic cancers $(1,2)$. The standard treatments for ovarian cancer patients are carboplatin and paclitaxel chemotherapy, which are effective in $80 \%$ of patients. However, almost all patients develop tumor recurrence and chemotherapy resistance (3). Furthermore, due to chemotherapy resistance, the 5-year survival rate for ovarian cancer patients is less than $45 \%$ (4). Therefore, novel therapeutic approaches are required to improve outcomes for ovarian cancer patients. Angiogenesis refers to the formation of new blood vessels from pre-existing endothelium, a physiological process that is vital for tissue repair and development $(5,6)$. In pathological states such as cancer, angiogenesis is a fundamental process that supplies tumors with oxygen and nutrition required for their growth and survival (Fig. 1). In addition, angiogenesis is an essential component of the metastatic cascade $(5,6)$. Vascular endothelial growth factor (VEGF) and its receptor (VEGFR) are major drivers of tumor angiogenesis. Inhibitors of VEGF and VEGFR are widely used for the treatment of solid tumors (7). Although angiogenesis-targeting therapies have shown promising clinical results in solid tumors, these therapies have shown only modest improvements in overall survival (8). In addition, it has been demonstrated that anti-angiogenic therapy results in worse clinical outcomes in patients who develop resistance to anti-angiogenic drugs, suggesting that the possible mechanism behind anti-angiogenic drug resistance may be the activation of alternative pathways of tumor neovascularization $(8,9)$.

Maniotis et al. observed tumor angiogenesis-independent vascular channels in highly aggressive uveal melanomas and termed the phenomenon vasculogenic mimicry (VM) (10). Since then, VM has been observed in many aggressive tumor types such as ovarian cancer, breast cancer, and glioma (11-13). VM is the formation of a nutrient and oxygen supply system by tumor cell-lined vessels, suggesting alternative pathways of tumor neovascularization (Fig. 1). Tumor cells associated with VM express markers associated with endothelial cells, the epithelial-mesenchymal transition (EMT), and cancer stem cells (CSCs) and contribute to the formation of the tumor vasculature (11).

EMT is a reversible phenotypic switching process by which 


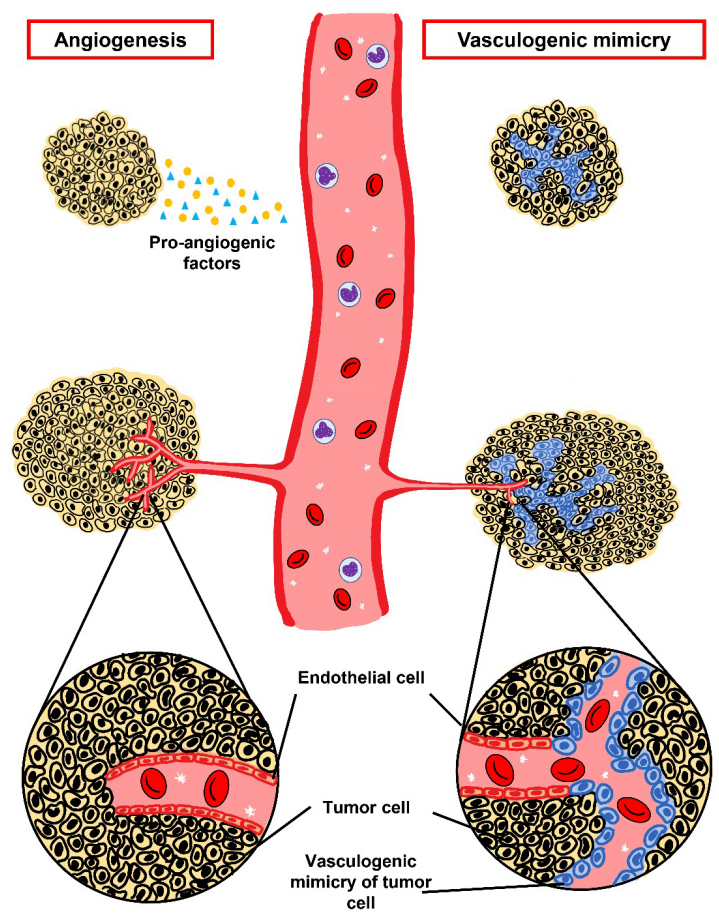

Fig. 1. Schematic representation of differences between angiogenesis and VM. During tumor angiogenesis, tumor cells secrete many pro-angiogenic factors to induce their vascularization via activation of pre-existing host endothelium, whereas VM is a tumor vascularization mechanism in which tumors create a blood supply system when faced with an insufficient supply of oxygen and nutrients.

epithelial cells lose their traits and acquire mesenchymal characteristics. Accumulating evidence suggests that activation of EMT not only triggers cancer cell invasion and metastasis, but also contributes to VM (14). CSCs are closely involved in recurrence and metastasis in various cancer cells and VM. It has been shown that cells positive for CSC markers such as CD133 and aldehyde dehydrogenase1 (ALDH1) play a key role in VM in various cancer cell types (15-18). VM is associated with advanced disease at presentation and an extremely poor prognosis in ovarian cancer $(12,16)$. Furthermore, it was shown that antiangiogenic therapy induces a hypoxic response and VM in ovarian cancer, suggesting that VM may serve as an alternative tumor neovascularization (19). In light of this evidence, further studies are urgently needed to understand the molecular mechanisms associated with VM in order to overcome the limitations of anti-angiogenic therapy and to develop novel therapeutics that target multiple cancers. In this review, we summarize the available knowledge regarding current anti-angiogenic therapies and the mechanisms involved in VM in ovarian cancer. Moreover, we discuss potential therapeutic strategies involving the regulation of VM in ovarian cancer.

\section{ANTI-ANGIOGENIC THERAPY IN OVARIAN CANCER}

Angiogenesis is a dynamic process that is essential for embryonic development, wound healing, and ovulation under physiological conditions and is tightly controlled by a balance between angiogenic stimuli and angiogenic inhibitors $(5,20)$. However, disruption of the balance between angiogenic and anti-angiogenic molecules can lead to pathological conditions such as cancer, diabetic microvascular complications, and inflammatory disorders $(5,20)$. Of known pro-angiogenic factors, the most well-known are members of the VEGF family such as VEGF-A, VEGF-B, VEGF-C, and VEGF-D. VEGF proteins promote angiogenesis by binding to VEGFR and subsequently activating signaling cascades (7). In addition, many studies have shown that VEGF signaling is highly activated in ovarian cancer where it is closely associated with tumor grade and poor prognosis (21-23). Thus, blocking pro-angiogenic signaling, such as VEGF signaling, may be an effective therapeutic option against ovarian cancer. Therapeutics that involve targeting anti-angiogenic drugs through VEGF/VEGFR signaling are summarized below.

\section{VEGF-A targeting agent: Bevacizumab}

Bevacizumab is the first recombinant humanized monoclonal antibody against VEGF-A to be recognized as an anti-angiogenic agent. Bevacizumab inhibits VEGF-A from binding to VEGFR. Bevacizumab is approved by the FDA for use in the treatment and maintenance of platinum-sensitive recurrent ovarian cancer. Bevacizumab, a representative molecular target drug, has proven clinical efficacy in the treatment of advanced and recurrent ovarian cancer whereby it blocks the development of new blood vessels in tumors, destroys existing blood vessels and reduces intratumoral pressure $(24,25)$. Use of bevacizumab by ovarian cancer patients delays tumor progression and is applied as a maintenance therapy against ovarian cancer. Recently, bevacizumab and chemotherapy have been combined for use in platinum-resistant ovarian cancer (26). However, bevacizumab has been shown to improve median progression-free survival (PFS) for 2-4 months (27). Thus, it is necessary to identify predictive biomarkers to enable achieving greater therapeutic responses to bevacizumab only or in combination with VM inhibitors to enhance the clinical outcomes of anti-angiogenic therapy in ovarian cancer patients.

VEGFR targeting agents: Cediranib, pazopanib, nintedanib Cediranib is an inhibitor of VEGFR (VEGFR1-3) tyrosine kinase which has been shown to have disappointing efficacy in many cancers and increased toxicity such as diarrhea, neutropenia, hypertension, and voice changes $(27,28)$. Nevertheless, cediranib treatment of ovarian cancer patients is promising, and significant improvements in progression-free survival have been identified in Phase 3 trials with chemotherapy and maintenance treatment $(29,30)$.

Pazopanib is a selective multi-targeted receptor tyrosine kinase inhibitor that inhibits VEGFR, platelet-derived growth 
factor receptor (PDGFR), c-KIT, c-Fms, and fibroblast growth factor receptor (FGFR), and tumor growth and angiogenesis $(31,32)$. Pazopanib also has side effects, such as neutropenia, fatigue, leucopenia, hypertension, and anemia (33). However, it has advantages in platinum-resistant and refractory treatment and platinum-sensitive maintenance in ovarian cancer (34, 35).

Nintedanib competitively inhibits non-receptor tyrosine kinases such as lymphocyte-specific protein tyrosine kinase (Lck), tyrosineprotein kinase Lyn (Lyn), proto-oncogene tyrosine-protein kinase Src (Src), and receptor tyrosine kinases such as PDGFR, FGFR, FLT3, and VEGFR (27). Nintedanib is used in a variety of diseases, sometimes in combination with other drugs for the treatment of some cancers (36). Nintedanib has common side effects including abdominal pain, vomiting, and diarrhea (37). However, Phase 3 trials have shown that treatments combining nintedanib with carboplatin and paclitaxel produce remarkable therapeutic effects in ovarian cancer patients. However, these positive effects were accompanied by serious side effects including adverse gastrointestinal events (38).

Angiogenesis inhibitors that do not target VEGF signaling are also used as ovarian cancer therapeutics. Angiopoietin 1 and 2 (Ang1/2) bind to the Tie-2 receptor and stimulate proliferation, motility, and survival in endothelial cells. Trebananib is a nonVEGF-dependent angiogenesis pathway inhibitor that binds to Ang $1 / 2$ and inhibits its action on the Tie- 2 receptor, thus inhibiting angiogenesis. Trebananib-based therapies have produced meaningful effects in terms of progression-free survival when combined with paclitaxel in patients with recurrent ovarian cancer (39).

\section{CLINICAL SIGNIFICANCE OF VM IN OVARIAN CANCER}

Anti-angiogenic therapy has some advantages over chemotherapy because it does not directly kill cancer cells but rather stops the formation of new blood vessels that rarely occur in tissues other than tumors. Therefore, it is well-tolerated by patients and is associated with fewer side effects. Unfortunately, the use of anti-angiogenic therapies results in a hypoxic tumor microenvironment and the development of resistance by alternative tumor vascularization (40). Understanding the mechanisms and signaling pathways involved in VM is expected to enable overcoming the limitations of anti-angiogenic therapies.

$\mathrm{VM}$ is a tumor cell-derived vascular network and a functional micro-circulation system that provides oxygen and nutrients to tumors independent of angiogenesis, which contributes to tumor growth and metastasis $(9,41)$. The first evidence of clinical significance of VM in ovarian cancer showed that VM was closely associated with shorter overall survival and aggressive tumor features (12). Since then, Yu et al. demonstrated that VM was significantly associated with tumor grade, tumor/lymph node/metastasis and advanced stage. Moreover, VM-positive ovarian cancer patients had significantly shorter overall survival compared with VM-negative ovarian cancer patients, suggesting that VM plays a crucial role in tumor progression and metastasis in ovarian cancer (15). Given that CSCs are involved in VM, it has been shown that a combination of markers of VM and the CSC marker CD133 was associated with advanced tumor stage, non-response to chemotherapy, and shorter overall survival time in ovarian cancer (16). Although VM is considered an alternative tumor vascularization mechanism for tumor invasion and metastasis and has been shown to be associated with poor clinical prognosis in ovarian cancer, few studies have assessed the molecular mechanisms associated with VM in ovarian cancer. Our efforts to understand the mechanism of VM in ovarian cancer may provide new insights to enable identifying novel therapeutic targets. The following sections will outline the current understanding of the molecular mechanisms associated with VM and will discuss the possibility of employing VM-targeted therapy in the treatment of ovarian cancer.

\section{MAJOR MECHANISMS OF VM IN OVARIAN CANCER}

Both tumor angiogenesis and VM are critical for tumor blood supply and play an essential role in the progression of tumor growth and metastasis. However, although signaling pathways governing tumor angiogenesis have been extensively studied $(5,7,20,42,43)$, signaling pathways associated with VM in ovarian cancer remain poorly understood. Nevertheless, owing to the importance of VM in cancer biology, the molecular mechanisms underlying VM have been gradually investigated in ovarian cancer. Given that VM occurs due to the ability of tumor cells to trans-differentiate into vascular endothelial-like phenotypes, the signaling pathways associated with vascular/angiogenesis and hypoxia-related signaling pathways have been investigated (Fig. 2).

\section{Vascular/angiogenesis signaling in VM}

Hendrix et al. investigated the biological significance of several endothelial cell markers such as CD31, Tie-1/2, and vascular endothelial (VE)-cadherin in VM, with the hypothesis that upregulation of endothelial cell markers in aggressive tumor cells may result in their ability to form VM. It was found that VE-cadherin was exclusively upregulated in highly aggressive melanoma cells, and knockdown of VE-cadherin led to inhibition of VM in aggressive melanoma cells, indicating an essential role for VE-cadherin in VM in melanoma cells (44). It has been demonstrated that VE-cadherin is involved in VM in ovarian cancer. VE-cadherin expression was highly upregulated in VM-positive patients and in SKOV3 and OVCAR3 ovarian cancer cells under hypoxic conditions (45). It has also been shown that VE-cadherin expression levels are closely associated with the propensity for VM in ovarian cancer cells and malignant ovarian cancer tissues $(46,47)$, suggesting biological significance of VE-cadherin in VM in malignant ovarian cancers.

VEGF is one of the most potent pro-angiogenic factors that 


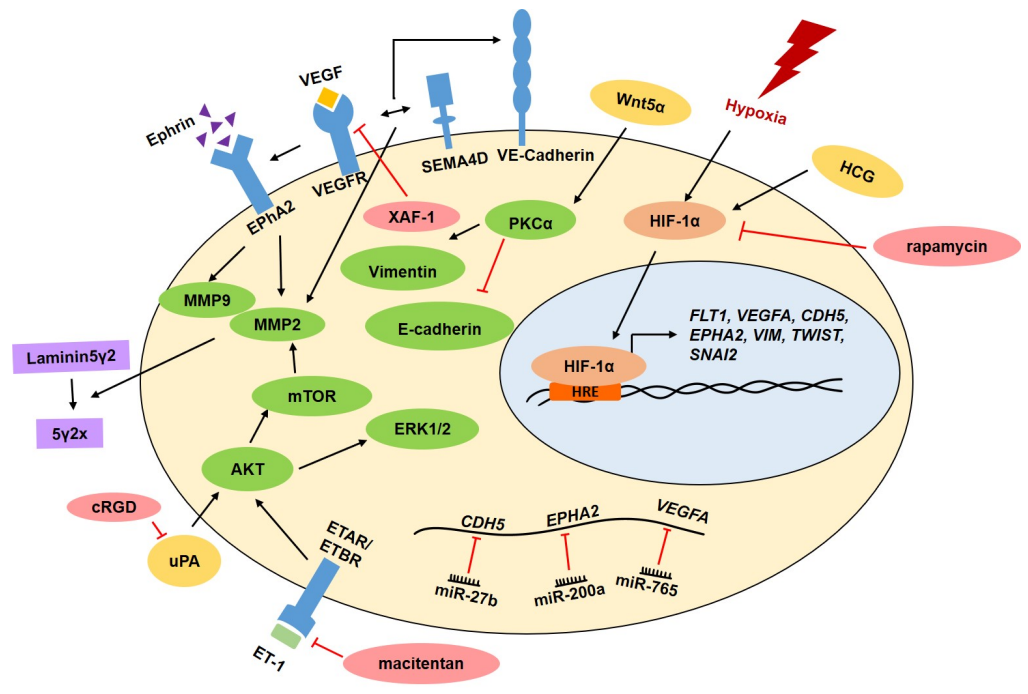

Fig. 2. Signaling pathways implicated in tumor cell VM. This figure summarizes the signaling pathways involved in the regulation of VM of ovarian cancer. Red solid bars denote VM inhibiting the signaling pathways, Black solid arrows denote VM promoting the signaling pathways.

play a key role in both physiological and pathological angiogenesis $(48,49)$. Several studies have identified potential roles of VEGF in VM in various cancer types (23, 50-52); however, the role of VEGF in VM remains controversial. The first study examining the role of VEGF-A in VM in ovarian cancer showed that VEGF-A promotes VM in vitro, and the expression of VEGF-A in VM-positive ovarian tumor tissues was higher than that in VM-negative ovarian tumor tissues. Mechanistic investigations revealed that VEGF-A stimulation induces the expression of EphA2, which is necessary for VEGF-induced neovascularization $(23,53)$, and knockdown of EphA2 inhibits VEGF-A-induced VM in ovarian cancer cells (23), suggesting that EphA2 plays a key role in VEGF-A-induced VM in ovarian cancer. Additionally, VEGF-A treatment markedly increased the activity of MMP9, and EphA2 knockdown resulting in decreased activity of MMP9 and MMP2 in SKOV3 and OVCAR3 ovarian cancer cells, respectively. These findings suggest that the VEGF-A/EphA2/MMP9 and MMP2 signaling axis plays an important role in the process of VM in ovarian cancer (23). Consistent with this report, VEGF and semaphorin4D (SEMA4D) expression levels were significantly higher in ovarian cancer tissues than in normal ovarian tissue, and the expression of VEGF positively correlated with the expression of SEMA4D in ovarian cancer tissues. Moreover, VEGF and SEMA4D showed synergistic effects on VM in the human ovarian cancer cell line A2780, and knockdown of VEGF and SEMA4D decreased the expression of VM-related molecues such as MMP2 and VE-cadherin in ovarian cancer cells (22). Conversely, another study showed that short-term bevacizumab treatment resulted in increased metastasis and induced VM in mice receiving short-term bevacizumab treatment, suggesting that VM may be an alternative mechanism underlying tumor resistance to anti-angiogenic therapy (19). The controversy surrounding the role of VEGF in the regulation of VM might reflect tumor cell type differences and tumor cell heterogeneity. Therefore, further studies are needed to fully elucidate the mechanisms associated with VEGF-driven VM and VEGF-independent VM.

\section{Hypoxia signaling in VM}

Tumor hypoxia arises due to rapid proliferation of cancer cells and dysfunctional tumor vasculature, resulting in rapid exhaustion of oxygen and nutrients. Tumor hypoxia is one of the most important features of the solid tumor microenvironment, playing a pivotal role in cancer development, malignancy, and progression (54). Under hypoxic conditions, hypoxia-inducible factor-1 (HIF-1) is the key transcription factor that binds to the hypoxia response element (HRE) to regulate the expression of hypoxiarelated molecues such as VEGF and EPO $(55,56)$, which allow tumor cells to adapt to a hostile microenvironment. Accumulating evidence suggests that tumor hypoxia plays a key role in cancer cell plasticity and is functionally essential for VM (45, $57,58)$. Potential hypoxia target molecues containing functional HREs such as VE-cadherin, VEGF-A, VEGFR-1, EphA2, and Twist influence VM (57). Initial investigation into the involvement of HIF-1 in VM in ovarian cancer showed that the HIF-1 $\alpha$ inhibitor rapamycin inhibits VM by suppressing CD31 and Factor VIII, suggesting the involvement of HIF-1 $\alpha$ in VM in ovarian cancer (59). Additionally, another study investigated the effects of hypoxia on VM and its underlying molecular mechanism(s) in ovarian cancer. HIF-1 $\alpha$ expression was correlated with expression of EMT markers such as vimentin, Twist1, and Slug as well as shorter survival in patients with VM-positive ovarian cancer. Under hypoxic conditions, ovarian cancer cells showed greater VM by inducing EMT. These findings indicate that EMT is important for hypoxia-induced VM in ovarian cancer (45). Hypoxia plays a key role in VM in ovarian cancer cells, and human chorionic gonadotropin (HCG) is a placental hormone that regulates placental angiogenesis 
during pregnancy which is ectopically expressed in many malignant tumors $(60,61)$. Su et al. investigated the involvement of HCG in VM in OVCAR3 ovarian cancer cells under hypoxic conditions. It was shown that hypoxia induced VM via upregulation of HCG expression, an effect that was abolished by knockdown of HIF-1 $\alpha$. In addition, HCG treatment led to significant VM and endothelial cell marker expression, even under normoxic conditions (62). By further exploring the role of HCG in VM in ovarian cancer in vivo, researchers showed that injection of OVCAR3 ovarian cancer cells overexpressing chorionic gonadotropin beta polypeptide 5 (CGB5) induced xenograft tumor formation and VM via upregulation of endothelial markers such as CD31, VEGF, and factor VIII (63). Considering a number of studies underscore the importance of hypoxia in VM for a variety of cancers, clarifying the underlying mechanisms associated with hypoxia-induced VM and establishing strategies to regulate VM are urgently needed.

\section{Other important factors in VM}

It has been reported that other genes are also involved in VM in ovarian cancer. X-linked inhibitor of apoptosis-associated factor 1 (XAF1) plays a key role in the regulation of apoptosis and angiogenic properties of endothelial cells and cancer cells $(64,65)$. Given that angiogenesis and apoptosis signaling are involved in VM $(53,66)$, Wang et al. investigated the potential role of XAF1 in VM in ovarian cancer and found that XAF1 expression was downregulated in the VM-positive group which was associated with high-grade advanced ovarian cancer. Overexpression of XAF1 decreased VEGF expression and inhibited the motility of SKOV3 ovarian cancer cells. An inhibitory role for XAF1 in relation to VM and VEGF expression was also shown in xenograft models, suggesting that XAF1 acts as a negative regulator of $\mathrm{VM}$ via VEGF inhibition (67).

Another regulator of VM is the Wnt family of proteins, which are key regulators of tumorigenesis, endothelial differentiation, and vascular development. The Wnt signaling pathway includes the $\beta$-Catenin-dependent (canonical) and -independent (non-canonical) pathways. Wnt5a, a member of the non-canonical Wnt family, is essential for cancer initiation and progression (68, 69). Qi et al. investigated the association between Wnt5a and VM in ovarian cancer and demonstrated that the expression of Wnt5a is significantly correlated with VM and protein kinase $\mathrm{C} \alpha(\mathrm{PKC} \alpha)$ expression, suggesting that it may act as a downstream factor of non-canonical Wnt5a signaling. An in vitro mechanistic investigation showed that Wnt5a induces VM and regulates the expression of EMT-related markers such as vimentin and E-cadherin in ovarian cancer cells. Additionally, it was shown that Wnt5a-induced EMT and VM are mediated by the PKC $\alpha$ pathway in ovarian cancer cells (70).

Decomposition of the extracellular matrix $(\mathrm{ECM})$ is an important process in EMT-induced VM. It has been shown that urokinase plasminogen activator (UPA) regulates tumor angiogenesis by degrading ECM molecules such as laminin, fibronectin, and collagen (71). Tang et al. investigated the association between
UPA and VM in ovarian cancer. VM occurrence was positively correlated with high uPA expression in 90 ovarian cancer cases and ovarian cancer cells, and uPA knockdown led to a decrease in VM via regulation of the AKT/mTOR/MMP2/ Laminin $5 \gamma 2$ signaling pathway in ovarian cancer cells. In addition, it was found that cyclin RGD (CRGD) inhibited VM in ovarian cancer cells via downregulation of uPA expression and EMT repression (72). These findings suggest that modulation of uPA expression may be a potential therapeutic target for VM in ovarian cancer.

Given that activation of endothelin-1 (ET-1)/endothelin A receptor (ETAR) and ET-1/ETBR signaling is closely associated with tumor growth, angiogenesis, metastasis, and chemoresistance, Sestito et al. investigated the role of ET-1 signaling in VM in ovarian cancer. It was demonstrated that the dual ETAR/ETBR antagonist macitentan significantly inhibited VM, and enabled inhibition of ET-1-induced activation of AKT and MAPK signaling pathways in cisplatin resistant A2780 and 2008 cells (73). Thus, macitentan could be a novel potential drug for ovarian cancer therapy, due to its ability to inhibit VM.

A recent study showed that syndecan-1 (SDC1) and tumor angiogenic-associated B-fibronectin isoform (B-FN) play a pivotal role in VM in ovarian cancer. Combined treatment with L19-IL2 (an immunocytokine specific for B-FN) and an anti-SDC1 46F2SIP (small immunoprotein) antibody was effective in reducing the expression of EMT markers and loss of cancer stemness traits, which were correlated with inhibition of VM in mice (74).

\section{MicroRNAs in VM}

MicroRNAs (miRNAs) are 21-25 nucleotides small non-coding RNAs which regulate gene expression post-transcriptionally. They recognize the 3 '-untranslated region ( $3^{\prime}$-UTR) of the target mRNA to promote degradation of the mRNA or to inhibit protein synthesis, thus reducing the expression of the target gene (75). Many studies have shown that miRNA expression is dysregulated in various diseases (76-78). Several studies have demonstrated that miRNAs play an important role in the regulation of VM in ovarian cancer $(46,79-81)$.

The miR-200 family (miR-141, miR-200a, miR-200b, miR-200c, and miR-429) is known to target transforming growth factor- $\beta$ (TGF- $\beta$ ), a potent inducer of EMT, and EMT-related transcription factors Zinc Finger E-Box Binding Homeobox (ZEB)-1 and $Z E B-2$, resulting in the inhibition of ovarian cancer invasion, migration, metastasis, and EMT (82). Given the inhibitory effect of the miR-200 family on the EMT process, Sun et al. investigated the role of miR-200a in VM in ovarian cancer. Expression of miR-200a was downregulated in VM-positive ovarian cancer, which is associated with high tumor grade/stage, and metastasis. A mechanistic study revealed that miR-200a inhibits VM by directly targeting EphA2, a key regulator of VM in ovarian cancer. These findings suggest that miR-200a-mediated EphA2 regulation plays important roles in the progression 
and prognosis of ovarian cancer patients (79).

As described above, VE-cadherin is highly overexpressed in VM-forming cancer cells and plays an essential role in VM in malignant ovarian cancer (45). Therefore, VE-cadherin-targeting miRNAs could be excellent targets for the control of VM in ovarian cancer. miR-27b plays an important role in the regulation of VM in ovarian cancer by directly targeting VE-cadherin. Low levels of miR-27b expression are strongly correlated with high levels of VE-cadherin expression and robust VM in ovarian cancer cells. It was demonstrated that miR-27b directly targets the 3'-UTR of VE-cadherin, and overexpression of miR$27 \mathrm{~b}$ inhibits $\mathrm{VM}$, migration, and invasion in ovarian cancer cells. Additionally, it was shown that miR-27b suppresses ovarian cancer-associated VM in vivo using a matrigel plug assay (46).

In another study, the authors investigated the relevance of hypoxia-responsive miRNAs to VM in SKOV3 ovarian cancer cells. It was shown that miR-765 was markedly downregulated under hypoxic conditions, and ectopic restoration of miR-765 significantly inhibited VM without affecting cell viability. Interestingly, it was identified that miR-765 directly targets VEGF, another key regulator in VM in ovarian cancer cells (80). Although our current understanding of the molecular mechanisms underlying VM in ovarian cancer is advancing, future studies are needed to fully understand the molecular mechanism(s) associated with VM in ovarian cancer. In particular, it has been demonstrated that CSCs are involved in VM in many cancers. Therefore, it will be important to determine the molecular mechanisms associated with VM in the context of ovarian CSCs in future studies. Fig. 2 shows a summary of the major signaling mechanisms involved in the regulation of VM in ovarian cancer.

\section{CONCLUSIONS}

Ovarian cancer is one of the most common forms of gynecologic cancer with an extremely high morbidity and mortality rate. Although initial responses to platinum and taxane-based chemotherapy are generally good, the recurrence rate is extremely high $(\sim 80 \%)$. Several studies show that anti-angiogenic therapy alone or in combination with chemotherapy is a promising therapeutic strategy for ovarian cancer. However, curative rates of anti-angiogenic therapy are not increased in most ovarian cancer patients (83), and anti-angiogenic therapy with bevacizumab induces a hypoxic response and VM (19), which may be associated with limited efficacy and poor therapeutic response to anti-angiogenic therapy in ovarian cancer. In addition, accumulating evidence suggests that VM is closely associated with poor prognosis, high metastatic potential, and shorter survival in cancer patients. Therefore, as an alternative tumor vascularization mechanism, VM targeting may represent a promising treatment option for ovarian cancer patients to enhance the clinical outcomes of anti-angiogenic therapy. In addition, a better understanding of the molecular mechanisms involved in VM will facilitate overcoming disad- vantages associated with current ovarian cancer therapeutics.

\section{ACKNOWLEDGEMENTS}

This research was supported by the Basic Science Research Program through the National Research Foundation of Korea (NRF) funded by the minister of Education, Science and Technology (NRF-2016R1A5A1011974 and NRF-2019R1A2C 4069815 to J.K., 2017R1D1A1B03034206 to J.G.C).

\section{CONFLICTS OF INTEREST}

The authors have no conflicting interests.

\section{REFERENCES}

1. Siegel RL, Miller KD and Jemal A (2019) Cancer statistics, 2019. CA Cancer J Clin 69, 7-34

2. Sudo T (2012) Molecular-targeted therapies for ovarian cancer: prospects for the future. Int J Clin Oncol 17, 424429

3. Hennessy BT, Coleman RL and Markman M (2009) Ovarian cancer. Lancet 374, 1371-1382

4. Webb PM and Jordan SJ (2017) Epidemiology of epithelial ovarian cancer. Best Pract Res Clin Obstet Gynaecol 41, 3-14

5. Park JA and Kwon YG (2018) Hippo-YAP/TAZ signaling in angiogenesis. BMB Rep 51, 157-162

6. Folkman J (2002) Role of angiogenesis in tumor growth and metastasis. Semin Oncol 29, 15-18

7. Shibuya M (2008) Vascular endothelial growth factordependent and -independent regulation of angiogenesis. BMB Rep 41, 278-286

8. Ribatti D, Annese T, Ruggieri S, Tamma R and Crivellato E (2019) Limitations of Anti-Angiogenic Treatment of Tumors. Transl Oncol 12, 981-986

9. Dunleavey JM and Dudley AC (2012) Vascular Mimicry: Concepts and Implications for Anti-Angiogenic Therapy. Curr Angiogenes 1, 133-138

10. Maniotis AJ, Folberg R, Hess A et al (1999) Vascular channel formation by human melanoma cells in vivo and in vitro: vasculogenic mimicry. Am J Pathol 155, 739-752

11. Zhang X, Zhang J, Zhou H, Fan G and Li Q (2019) Molecular Mechanisms and Anticancer Therapeutic Strategies in Vasculogenic Mimicry. J Cancer 10, 6327-6340

12. Sood AK, Fletcher MS, Zahn CM et al (2002) The clinical significance of tumor cell-lined vasculature in ovarian carcinoma: implications for anti-vasculogenic therapy. Cancer Biol Ther 1, 661-664

13. Park Y and Kim J (2019) Regulation of IL- 6 signaling by miR-125a and let-7e in endothelial cells controls vasculogenic mimicry formation of breast cancer cells. BMB Rep 52, 214-219

14. Fan YL, Zheng M, Tang YL and Liang XH (2013) A new perspective of vasculogenic mimicry: EMT and cancer stem cells (Review). Oncol Lett 6, 1174-1180

15. Yu L, Zhu B, Wu S et al (2017) Evaluation of the correlation of vasculogenic mimicry, ALDH1, KiSS-1, and 
MACC1 in the prediction of metastasis and prognosis in ovarian carcinoma. Diagn Pathol 12, 23

16. Liang J, Yang B, Cao Q and Wu X (2016) Association of Vasculogenic Mimicry Formation and CD133 Expression with Poor Prognosis in Ovarian Cancer. Gynecol Obstet Invest 81, 529-536

17. Chae YC and Kim JH (2018) Cancer stem cell metabolism: target for cancer therapy. BMB Rep 51, 319-326

18. Zhang D, Sun B, Zhao X et al (2014) Twist1 expression induced by sunitinib accelerates tumor cell vasculogenic mimicry by increasing the population of CD133+ cells in triple-negative breast cancer. Mol Cancer 13, 207

19. Xu Y, Li Q, Li XY, Yang QY, Xu WW and Liu GL (2012) Short-term anti-vascular endothelial growth factor treatment elicits vasculogenic mimicry formation of tumors to accelerate metastasis. J Exp Clin Cancer Res 31, 16

20. Carmeliet $P$ (2005) Angiogenesis in life, disease and medicine. Nature 438, 932-936

21. Boocock CA, Charnock-Jones DS, Sharkey AM et al (1995) Expression of vascular endothelial growth factor and its receptors flt and KDR in ovarian carcinoma. J Natl Cancer Inst 87, 506-516

22. Chen Y, Zhang L, Liu WX and Wang K (2018) VEGF and SEMA4D have synergistic effects on the promotion of angiogenesis in epithelial ovarian cancer. Cell Mol Biol Lett 23, 2

23. Wang JY, Sun T, Zhao XL et al (2008) Functional significance of VEGF-a in human ovarian carcinoma: role in vasculogenic mimicry. Cancer Biol Ther 7, 758-766

24. Reinthaller A (2016) Antiangiogenic therapies in ovarian cancer. Memo 9, 139-143

25. Markowska A, Sajdak S, Markowska J and Huczynski A (2017) Angiogenesis and cancer stem cells: New perspectives on therapy of ovarian cancer. Eur J Med Chem 142, 87-94

26. Richardson DL (2019) New and Novel Therapies for Gynecologic Cancers. Semin Oncol Nurs 35, 217-219

27. Cortez AJ, Tudrej P, Kujawa KA and Lisowska KM (2018) Advances in ovarian cancer therapy. Cancer Chemother Pharmacol 81, 17-38

28. Wedge SR, Kendrew J, Hennequin LF et al (2005) AZD2171: a highly potent, orally bioavailable, vascular endothelial growth factor receptor-2 tyrosine kinase inhibitor for the treatment of cancer. Cancer Res 65, 4389-4400

29. Ledermann JA, Embleton AC, Raja F et al (2016) Cediranib in patients with relapsed platinum-sensitive ovarian cancer (ICON6): a randomised, double-blind, placebo-controlled phase 3 trial. Lancet 387, 1066-1074

30. Raja FA, Griffin CL, Qian W et al (2011) Initial toxicity assessment of ICON6: a randomised trial of cediranib plus chemotherapy in platinum-sensitive relapsed ovarian cancer. Br J Cancer 105, 884-889

31. Pick AM and Nystrom KK (2012) Pazopanib for the treatment of metastatic renal cell carcinoma. Clin Ther 34, 511-520

32. Schoffski P (2012) Pazopanib in the treatment of soft tissue sarcoma. Expert Rev Anticancer Ther 12, 711-723

33. Pignata S, Lorusso D, Scambia G et al (2015) Pazopanib plus weekly paclitaxel versus weekly paclitaxel alone for platinum-resistant or platinum-refractory advanced ovarian cancer (MITO 11): a randomised, open-label, phase 2 trial. Lancet Oncol 16, 561-568

34. du Bois A, Floquet A, Kim JW et al (2014) Incorporation of pazopanib in maintenance therapy of ovarian cancer. J Clin Oncol 32, 3374-3382

35. Floquet A, Vergote I, Colombo $\mathrm{N}$ et al (2015) Progressionfree survival by local investigator versus independent central review: comparative analysis of the AGO-OVAR16 Trial. Gynecol Oncol 136, 37-42

36. Awasthi N and Schwarz RE (2015) Profile of nintedanib in the treatment of solid tumors: the evidence to date. Onco Targets Ther 8, 3691-3701

37. Hughes $\mathrm{G}$, Toellner $\mathrm{H}$, Morris $\mathrm{H}$, Leonard $\mathrm{C}$ and Chaudhuri N (2016) Real World Experiences: Pirfenidone and Nintedanib are Effective and Well Tolerated Treatments for Idiopathic Pulmonary Fibrosis. J Clin Med 5, 78-89

38. du Bois A, Kristensen G, Ray-Coquard I et al (2016) Standard first-line chemotherapy with or without nintedanib for advanced ovarian cancer (AGO-OVAR 12): a randomised, double-blind, placebo-controlled phase 3 trial. Lancet Oncol 17, 78-89

39. Monk BJ, Poveda A, Vergote I et al (2014) Anti-angiopoietin therapy with trebananib for recurrent ovarian cancer (TRINOVA-1): a randomised, multicentre, double-blind, placebo-controlled phase 3 trial. Lancet Oncol 15, 799-808

40. van Beijnum JR, Nowak-Sliwinska P, Huijbers EJ, Thijssen VL and Griffioen AW (2015) The great escape; the hallmarks of resistance to antiangiogenic therapy. Pharmacol Rev 67, 441-461

41. Sood AK, Seftor EA, Fletcher MS et al (2001) Molecular determinants of ovarian cancer plasticity. Am J Pathol $158,1279-1288$

42. Jo HN, Kang H, Lee A et al (2017) Endothelial miR-26a regulates VEGF-Nogo-B receptor-mediated angiogenesis. BMB Rep 50, 384-389

43. Carmeliet $P$ and Jain RK (2011) Molecular mechanisms and clinical applications of angiogenesis. Nature 473, 298307

44. Hendrix MJ, Seftor EA, Meltzer PS et al (2001) Expression and functional significance of VE-cadherin in aggressive human melanoma cells: role in vasculogenic mimicry. Proc Natl Acad Sci U S A 98, 8018-8023

45. Du J, Sun B, Zhao X et al (2014) Hypoxia promotes vasculogenic mimicry formation by inducing epithelialmesenchymal transition in ovarian carcinoma. Gynecol Oncol 133, 575-583

46. Liu W, Lv C, Zhang B, Zhou Q and Cao Z (2017) MicroRNA-27b functions as a new inhibitor of ovarian cancer-mediated vasculogenic mimicry through suppression of VE-cadherin expression. RNA 23, 1019-1027

47. Czekierdowski A, Czekierdowska S, Stachowicz N, Lozinski T and Gurynowicz G (2017) Mig-7 expression and vasculogenic mimicry in malignant ovarian tumors. Ginekol Pol 88, 552-561

48. Lapeyre-Prost A, Terme M, Pernot S et al (2017) Immunomodulatory Activity of VEGF in Cancer. Int Rev Cell Mol Biol 330, 295-342

49. Jeong MS, Bae JS and Jin HK (2019) Vascular endothelial growth factor improves the therapeutic effects of cyclodextrin in Niemann-Pick type C mice. Anim Cells Syst (Seoul) 


\section{3, 346-354}

50. Mei J, Gao Y, Zhang L et al (2008) VEGF-siRNA silencing induces apoptosis, inhibits proliferation and suppresses vasculogenic mimicry in osteosarcoma in vitro. Exp Oncol 30, 29-34

51. Qin L, Ren Y, Chen AM et al (2014) Peroxisome proliferator-activated receptor gamma ligands inhibit VEGF-mediated vasculogenic mimicry of prostate cancer through the AKT signaling pathway. Mol Med Rep 10, 276-282

52. Cong R, Sun Q, Yang L, Gu H, Zeng $Y$ and Wang B (2009) Effect of Genistein on vasculogenic mimicry formation by human uveal melanoma cells. J Exp Clin Cancer Res 28, 124

53. Cheng N, Brantley DM, Liu H et al (2002) Blockade of EphA receptor tyrosine kinase activation inhibits vascular endothelial cell growth factor-induced angiogenesis. Mol Cancer Res 1, 2-11

54. Manandhar S and Lee YM (2018) Emerging role of RUNX3 in the regulation of tumor microenvironment. BMB Rep 51, 174-181

55. Zimna A and Kurpisz M (2015) Hypoxia-Inducible Factor-1 in Physiological and Pathophysiological Angiogenesis: Applications and Therapies. Biomed Res Int 2015, 549412

56. Yu K, Xiang L, Li S, Wang S, Chen C and Mu H (2019) HIF1alpha promotes prostate cancer progression by increasing ATG5 expression. Anim Cells Syst (Seoul) 23, 326-334

57. Zhang S, Zhang D and Sun B (2007) Vasculogenic mimicry: current status and future prospects. Cancer Lett 254, 157-164

58. Sun B, Zhang D, Zhang S, Zhang W, Guo $\mathrm{H}$ and Zhao X (2007) Hypoxia influences vasculogenic mimicry channel formation and tumor invasion-related protein expression in melanoma. Cancer Lett 249, 188-197

59. Su M, Feng YJ, Yao LQ et al (2008) Plasticity of ovarian cancer cell SKOV3ip and vasculogenic mimicry in vivo. Int J Gynecol Cancer 18, 476-486

60. Herr F, Baal N, Reisinger K et al (2007) HCG in the regulation of placental angiogenesis. Results of an in vitro study. Placenta 28 Suppl A, S85-93

61. Muller CY and Cole LA (2009) The quagmire of hCG and hCG testing in gynecologic oncology. Gynecol Oncol $112,663-672$

62. Su M, Wei W, Xu X et al (2011) Role of hCG in vasculogenic mimicry in OVCAR-3 ovarian cancer cell line. Int J Gynecol Cancer 21, 1366-1374

63. Gao S, Fan C, Huang H, Zhu C, Su M and Zhang Y (2016) Effects of HCG on human epithelial ovarian cancer vasculogenic mimicry formation in vivo. Oncol Lett 12 , 459-466

64. Qiao L, Gu Q, Dai Y et al (2008) XIAP-associated factor 1 (XAF1) suppresses angiogenesis in mouse endothelial cells. Tumour Biol 29, 122-129

65. Zhu LM, Shi DM, Dai Q et al (2014) Tumor suppressor XAF1 induces apoptosis, inhibits angiogenesis and inhibits tumor growth in hepatocellular carcinoma. Oncotarget 5, 5403-5415

66. Vartanian AA, Burova OS, Stepanova EV and Baryshnikov
AY (2007) The involvement of apoptosis in melanoma vasculogenic mimicry. Melanoma Res 17, 1-8

67. Wang Y, Liu P, Wang X and Mao H (2017) Role of Xlinked inhibitor of apoptosisassociated factor1 in vasculogenic mimicry in ovarian cancer. Mol Med Rep $16,325-330$

68. Dejana $E$ (2010) The role of wnt signaling in physiological and pathological angiogenesis. Circ Res 107, 943-952

69. Kim NH, Lee Y and Yook JI (2018) Dishevelling Wnt and Hippo. BMB Rep 51, 425-426

70. Qi H, Sun B, Zhao X et al (2014) Wnt5a promotes vasculogenic mimicry and epithelial-mesenchymal transition via protein kinase Calpha in epithelial ovarian cancer. Oncol Rep 32, 771-779

71. Rabbani SA and Mazar AP (2001) The role of the plasminogen activation system in angiogenesis and metastasis. Surg Oncol Clin N Am 10, 393-415, x

72. Tang J, Wang J, Fan L et al (2016) cRGD inhibits vasculogenic mimicry formation by down-regulating UPA expression and reducing EMT in ovarian cancer. Oncotarget 7, 24050-24062

73. Sestito R, Cianfrocca R, Rosano L et al (2016) Macitentan blocks endothelin-1 receptor activation required for chemoresistant ovarian cancer cell plasticity and metastasis. Life Sci 159, 43-48

74. Orecchia P, Balza E, Pietra G et al (2019) L19-IL2 Immunocytokine in Combination with the Anti-Syndecan-1 46F2SIP Antibody Format: A New Targeted Treatment Approach in an Ovarian Carcinoma Model. Cancers (Basel) 11, 1232-1250

75. Kim J (2018) MicroRNAs as critical regulators of the endothelial to mesenchymal transition in vascular biology. BMB Rep 51, 65-72

76. Hernandez R, Sanchez-Jimenez E, Melguizo C, Prados and Rama AR (2018) Downregulated microRNAs in the colorectal cancer: diagnostic and therapeutic perspectives. BMB Rep 51, 563-571

77. Suh N (2018) MicroRNA controls of cellular senescence. BMB Rep 51, 493-499

78. Kinose Y, Sawada K, Nakamura K and Kimura T (2014) The role of microRNAs in ovarian cancer. Biomed Res Int 2014, 249393

79. Sun Q, Zou X, Zhang T, Shen J, Yin Y and Xiang J (2014) The role of miR-200a in vasculogenic mimicry and its clinical significance in ovarian cancer. Gynecol Oncol 132, 730-738

80. Salinas-Vera YM, Gallardo-Rincon D, Garcia-Vazquez R et al (2019) HypoxamiRs Profiling Identify miR-745 as a Regulator of the Early Stages of Vasculogenic Mimicry in SKOV3 Ovarian Cancer Cells. Front Oncol 9, 381

81. Cao L, Wan Q, Li F and Tang CE (2018) MiR-363 inhibits cisplatin chemoresistance of epithelial ovarian cancer by regulating snail-induced epithelial-mesenchymal transition. BMB Rep 51, 456-461

82. Choi PW and Ng SW (2017) The Functions of MicroRNA200 Family in Ovarian Cancer: Beyond Epithelial-Mesenchymal Transition. Int J Mol Sci 18, 1207-1226

83. Monk BJ, Minion LE and Coleman RL (2016) Antiangiogenic agents in ovarian cancer: past, present, and future. Ann Oncol 27 Suppl 1, i33-i39 